\title{
Manual immature reticulocyte fraction; a surrogate marker to assess post traumatic blood loss
}

\author{
Wickramaratne KAC ${ }^{1}$, Wijegunawardena $\mathrm{JKD}^{2}$, Wijewickrama DC $^{3}$ \\ ${ }^{\prime}$ Department of Pathology, ${ }^{3}$ Department of Physiology, Faculty of Medicine, University of Ruhuna, Galle, Sri Lanka. \\ ${ }^{2}$ Faculty of Allied Health Sciences, University of Ruhuna, Galle, Sri Lanka.
}

Correspondence: Dr. Chandana Wickramaratne

e-mail:kacw@med.ruh.ac.lk

D https://orcid.org/0000-0003-4394-2535

Submitted on 24.10 .2021 and accepted for publication on 04.12.2021

\begin{abstract}
Introduction: Erythropoietic response following chemotherapy and stem cell transplant is assessed by reticulocytosis and immature reticulocyte fraction (IRF). They are not used to assess erythropoiesis following acute haemorrhage even though it is known to cause reticulocytosis. This study was performed to evaluate the use of IRF as a marker of degree of post-traumatic haemorrhage.
\end{abstract}

Methods: A cross sectional descriptive study was performed using routine anticoagulated blood samples collected from patients admitted with acute traumatic haemorrhage to an emergency treatment unit. Differential and total reticulocyte counts were performed manually using standard techniques with bright field microscopy. The data on severity of blood loss were extracted from clinical records.

Results: There were total of 38 patients. Mean IRF of patients with mild $(n=14)$, moderate $(n=19)$ and severe $(n=05)$ haemorrhage were $1.86 \pm 1.0 \%, 3.26 \pm 1.8 \%$ and $10.2 \pm 3.6 \%$ respectively. Mean manual IRF of group with severe haemorrhage was significantly higher than those of all the others $(p=0.000)$. The mean manual IRF of moderate haemorrhage group was significantly higher than that of mild group $(p=0.01)$. Manual IRF and severity of haemorrhage were significantly positively correlated $(\mathrm{r}=0.71, p<0.01, \mathrm{n}=38)$. Immature reticulocytes of stage I was observed only in patients with severe haemorrhage.

Conclusion: The manual IRF can be used as a reliable marker of severity of post-traumatic acute haemorrhage.

Keywords: Acute haemorrhage, surrogate marker, immature reticulocyte fraction, post-traumatic blood loss, Reticulocytes, manual immature reticulocyte fraction.

\section{Introduction}

Post traumatic haemorrhage needs prompt medical attention as it can cause significant morbidity and mortality due to acute hypovolaemia and shock (1). Degree of blood loss is classified based on clinical assessment in acute trauma care. Following haemorrhage, erythropoiesis is increased in the marrow and more immature forms of red cells (reticulocytes) are rapidly released in to the peripheral circulation. Reticulocytes can be classified based on their maturation using both manual and automated techniques (2-4). The immature reticulocyte fraction (IRF), is a novel parameter available in fully automated haematology analysers. It is used as an early indicator of marrow erythropoietic activity in stress conditions and to assess marrow response following therapy or stem cell transplant (5-7). Automated IRF is prohibitively costly to use in routine care in limited resource settings $(8-10)$. 
Manual reticulocyte counting is performed in many limited resource laboratories but manual IRF calculation is not. Therefore, this study was carried out to evaluate the possibility of using manual IRF in assessing the degree of haemorrhage in acute trauma.

\section{Methods}

All the patients admitted to an emergency treatment unit (ETU) of a Teaching Hospital during a period of one month, with post-traumatic acute haemorrhage irrespective of the degree of haemorrhage were recruited to the study. Informed written consent was taken from the patient when he or she was in a position to do so and when they were unable to consent, assent was obtained from a guardian or relative. Those who had known clinical conditions that could directly affect haematological results were excluded from the study.

Clinical assessment of severity of blood loss on admission and the time taken for admission since traumatic event were extracted from patient records which were maintained by acute trauma physicians.

Venous blood samples $(5 \mathrm{ml})$ collected from antecubital fossa using standard techniques \{in to tubes containing Dipotassium Ethyle-nediaminetetraacetic acid (K2EDTA) \} were used to prepare blood smears to perform reticulocyte counting. Blood smears were prepared within one hour of collection of samples and reticulocyte staining was performed using standard supra vital staining procedure $(9,10)$. The quality of the stain was verified by the presence of stained platelets and white cells in each smear and it was considered the positive control. The differential count of immature reticulocytes and total reticulocyte count were performed manually using bright field microscope (X100 power/ oil immersion in Olympus CX 32) fitted with an ocular graticule. The IRF (stage I and II reticulocytes) was calculated as a percentage of all the reticulocytes counted. Reticulocytes were classified as per the Heilmeier classification (Figure 1) and the differential count of each stage of reticulocytes was performed (4). The reticulocyte counting and manual IRF calculations were performed in duplicates by the researchers.
A difference of $10 \%$ or less in the duplicate counts was considered acceptable. Reticulocyte percentages of all the patients were monitored hourly for four to five hours.

Data were analysed using MS-Excel 2010 and $R$-Studio statistical software. According to the clinical assessment on the patients' records the subjects were grouped in to mild, moderate and severe blood loss. The differences between groups were assessed using student's t-Test. The correlation was assessed with Spearman correlation coefficient. $p<0.05$ was considered significant.

Ethics approval was obtained from the Ethical Review Committee of Faculty of Allied Health Sciences, University of Ruhuna, Sri Lanka. Institutional permission and approval was obtained to retrieve data and to conduct the research in the emergency trauma unit, which was mandatory to the obtain ethical clearance.

\section{Results}

There were total of 38 patients who fulfilled the inclusion criteria and of them $24(63.1 \%)$ were males and $14(36.8 \%)$ were females. Of the total, 14 had mild, 19 had moderate and five had severe acute post traumatic haemorrhage. The median age of the study group was 36 years.

The mean manual IRF were $1.86 \pm 1.0 \%, 3.26 \pm$ $1.8 \%$ and $10.2 \pm 3.6 \%$ in patients with mild, moderate and severe haemorrhage respectively. The mean manual IRF of patients with severe haemorrhage was significantly higher than those of all the other patients with haemorrhage (mild and moderate) $(p=<0.001)$. The mean manual IRF of patients with moderate haemorrhage was also significantly higher than that of the patients with mild haemorrhage $(p=0.01)$. The manual IRF and the severity of haemorrhage were significantly positively correlated $(\mathrm{r}=.71, p<0.01$, $\mathrm{n}=38$ ) (Figure 2).

All four stages of reticulocytes were observed in patients with severe haemorrhage while only stages II, III and IV were observed in mild and moderate haemorrhages (Figure 3). 
Stage I

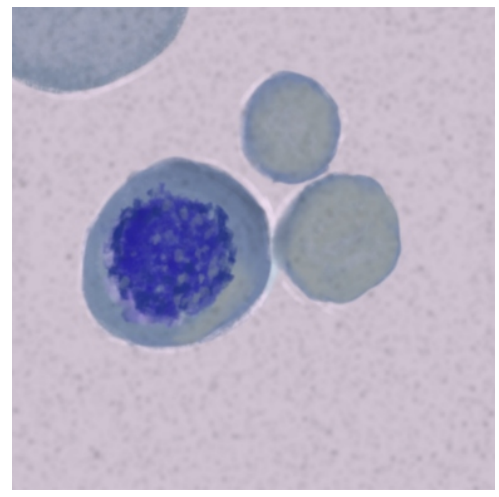

Stage III

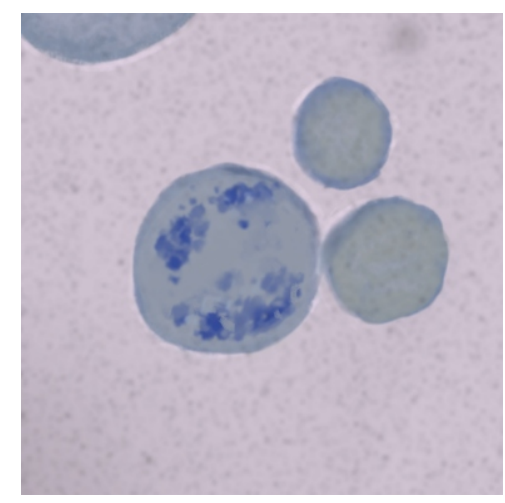

Stage II

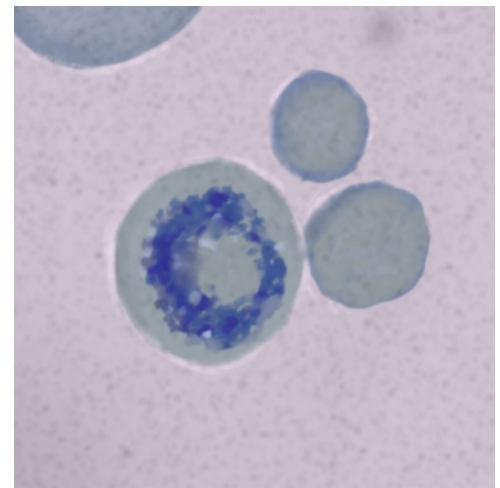

Stage IV

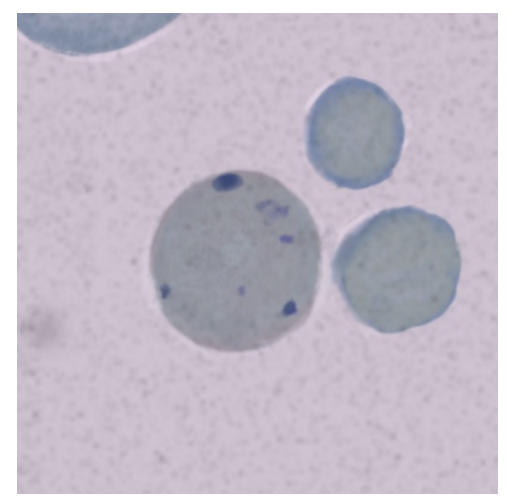

Figure 1: Maturation stages of reticulocytes according to Heilmeier classification. Stage I: non-nucleated red cells appearing with a dense clumped reticulum; Stage II: extended network of loose reticulum; Stage III; scattered granules with residual reticulum network; Stage IV: scattered granules

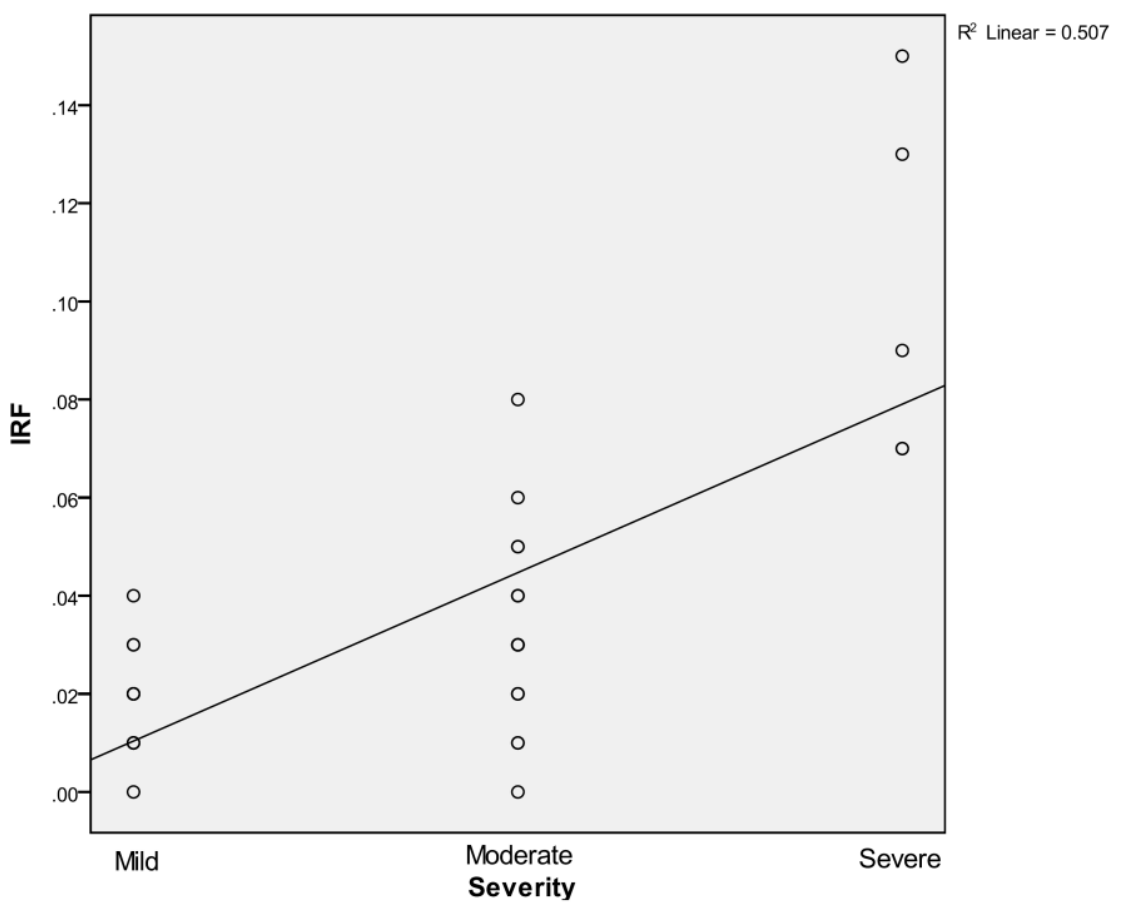

Figure 2: Correlation of immature reticulocyte fraction (IRF) and severity of haemorrhage 

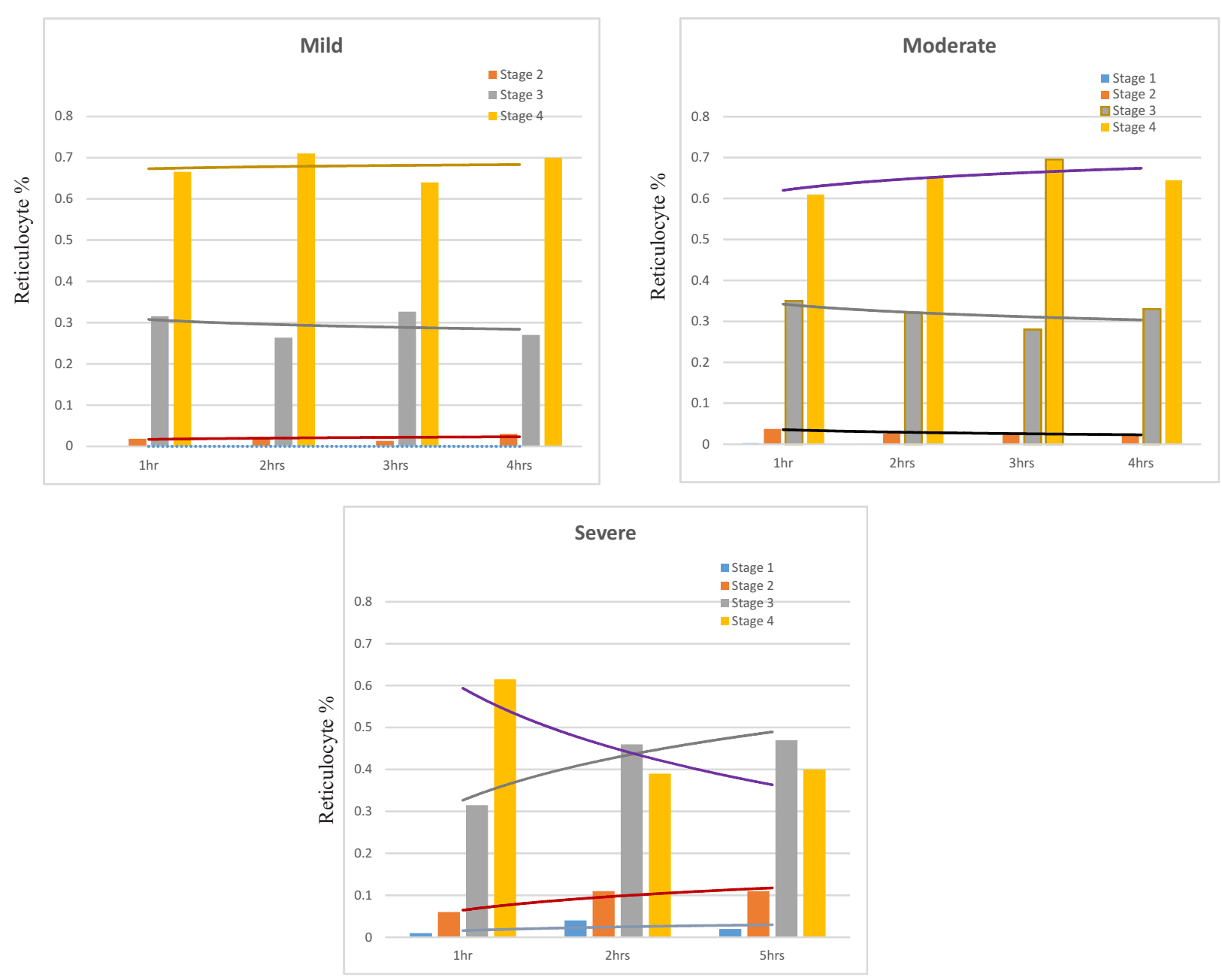

Figure 3: Percentages of reticulocyte stages and time duration since trauma in mild, moderate and severe haemorrhages and their trend lines

\section{Discussion}

Assessment of trauma-related blood loss is subjective and is supported mainly by clinical criteria (1). The subjective nature of this assessment is improved by the use of non-laboratory parameters such as blood pressure, pulse rate, pulse pressure, urine output and level of consciousness. The assessment of haemorrhagic shock is aided by laboratory parameters such as complete blood count and arterial blood gas analysis (10-14). Yet, accurate estimation of blood loss is subjective and challenging.

The automated IRF is considered one of the best parameters to detect early marrow response following chemotherapy or stem cell transplant (1-8). The data on use of automated or manual IRF to assess degree of haemorrhage in routine trauma care is not found in literature.
The peripheral blood reticulocyte count of healthy adults is $1 \%-2 \%$. All the reticulocytes in peripheral blood of healthy individuals belong to Heilmeier stages III and IV and are considered the mature forms $(4,8,9)$. The reticulocytes stages I and II (immature forms) are not normally found in peripheral circulation of healthy individuals (15). In this study, both stages I and II of immature reticulocytes were seen in peripheral blood of all the patients with severe haemorrhage. The patients with mild and moderate haemorrhage showed only the stage II of immature reticulocytes. Therefore, increased IRF (both stages I \& II) in peripheral blood appears to be an objective marker which differentiate severe haemorrhage from mild and moderate. Increased IRF of only stage II could be considered a marker of mild and moderate 
haemorrhage. In addition, stage IV reticulocytes showed progressive decline over five hours of study period in all the patients with severe haemorrhage.

None of the acute blood loss assessment tools have incorporated either automated or manual IRF for estimation of blood loss. The findings of this study show the value of estimation of either manual or possibly automated IRF as an objective surrogate indicator of degree of haemorrhage. Since, patients with moderate and severe bleeding are inevitably managed with crystalloids and/or blood transfusions; one can argue the reliability of IRF following an intervention. The details of standard care given to the patients before collection of blood samples for IRF were not collected in this study. However, irrespective of whether volume expanders were given or not, the patients with moderate and severe haemorrhages showed high IRF proving its applicability in detecting the degree of post traumatic acute haemorrhage. In addition, decrement of percentages of mature forms (stage IV) and increment of immature forms (stages I and II) appeared to be unique for severe haemorrhage.

Further studies are needed to assess the effects of volume resuscitation and other comorbid factors on IRF in post-traumatic acute haemorrhage and to demonstrate the reliability of IRF in estimation of degree of haemorrhage in non-traumatic or trauma related concealed (retroperitoneal, intraabdominal or intra muscular) haemorrhage.

\section{Limitations}

This study was carried out using manual method available for reticulocyte counting and it has its own limitations related to distribution of cells in smear, counting procedure and the limited number of cells counted. If the automated method was used, highly accurate and precise information on reticulocytes could have been obtained as large number of cells are counted in that method thus, it would have provided more accurate objective information on the relationship between IRF and the degree of acute blood loss in trauma. In this study, the grading of blood loss was performed by acute care physicians and surgeons and not by the researchers. If a universal grading scale for classifying degree of blood loss was incorporated in the assessment of blood loss, highly standardized information could have been gathered.

Manual IRF and progressive increment of IRF while decrement of percentages of mature reticulocytes reliably indicate severity of post traumatic acute haemorrhage and would be potentially useful even in assessment of acute concealed haemorrhage.

\section{Acknowledgements}

We acknowledge the administrative team of emergency treatment unit, Teaching Hospital, Karapitiya, Galle, Sri Lanka and staff of Medical Laboratory Science Department of Faculty of Allied Health Sciences for the provision of laboratory facilities to conduct this research project.

\section{Competing Interests:}

The authors have no competing interests.

\section{Funding:}

Authors received no funding from any institution or organization to carry out this study.

\section{References}

1. Spahn DR, Bouillon B, Cerny V, Duranteau J, Filipescu D, Hunt BJ, et al. The European guideline on management of major bleeding and coagulopathy following trauma: Fifth edition. Critical Care (London, England). 2019; 23(1): 98.

2. Piva E, Brugnara C, Spolaore F, Plebani M. Clinical utility of reticulocyte parameters. Clinics in Laboratory Medicine. 2015;35(1): 133-163.

3. Molina JR, Sanchez-Garcia J, Torres A, Alvarez MA, Serrano J, Casaño J, et al. Reticulocyte maturation parameters are reliable early predictors of hematopoietic engraftment after allogeneic stem cell transplantation. Biology of blood and marrow transplantation: Journal of the American Society for Blood and Marrow Transplantation. 2007; 13(2): 172-182.

4. Koepke JF, Koepke JA. Reticulocytes. Clinical and Laboratory Haematology. 1986; 8(3): 169-179. 
5. Morkis IV, Farias MG, Rigoni LD, Scotti L, Gregianin LJ, Daudt LE, et al. Assessment of immature platelet fraction and immature reticulocyte fraction as predictors of engraftment after haematopoietic stem cell transplantation. International Journal of Laboratory Haematology. 2015; 37(2): 259-264.

6. Md Noor, Sabariah and Leong, Cooi Fun, Cheong SK. Immature reticulocyte fraction in guiding stem cell harvest in autologous peripheral blood stem cell transplant. Malaysian Journal of Medicine and Health Sciences. 2014; 10: 1-6.

7. Raja-Sabudin RZ, Othman A, Ahmed-Mohamed KA, Ithnin A, Alauddin $\mathrm{H}$, Alias $\mathrm{H}$, et al. Immature reticulocyte fraction is an early predictor of bone marrow recovery post chemotherapy in patients with acute leukemia. Saudi Medical Journal. 2014;35(4): 346-349.

8. Buttarello M, Bulian P, Farina G, Petris MG, Temporin V, Toffolo L. Five fully automated methods for performing immature reticulocyte fraction: comparison in diagnosis of bone marrow aplasia. American Journal of Clinical Pathology. 2002; 117(6): 871-879.

9. Bain BJ, Bates I, Laffan MA. Dacie and Lewis Practical Haematology E-Book: Elsevier Health Sciences; 2016.

10. World Health Organization. Health Laboratory T, Blood Safety U, International Committee for Standardization in Haematology. Expert Panel in C. ICSH guidelines for reticulocyte counting by microscopy on supravitally stained preparations/ prepared on behalf of the World Health Organization by the Expert Panel on Cytometry of the International Council for Standardization in Haematology. Geneva: World Health Organization; 1992.
11. Figueiredo S, Taconet C, Harrois A, Hamada S, Gauss T, Raux M, et al. How useful are haemoglobin concentration and its variations to predict significant haemorrhage in the early phase of trauma? A multicentric cohort study. Annals of Intensive Care. 2018; 8(1): 76.

12. Gutierrez G, Reines HD, Wulf-Gutierrez ME. Clinical review: haemorrhagic shock. Critical Care (London, England). 2004; 8(5): 373-381.'

13. Hunt BJ, Allard S, Keeling D, Norfolk D, Stanworth SJ, Pendry K. A practical guideline for the haematological management of major haemorrhage. British Journal of Haematology. 2015; 170(6): 788-803.

14. Kauvar DS, Lefering R, Wade CE. Impact of haemorrhage on trauma outcome: an overview of epidemiology, clinical presentations, and therapeutic considerations. The Journal of Trauma. 2006; 60(6 Suppl): S3-11.

15. Crouch JY, Kaplow LS. Relationship of reticulocyte age to polychromasia, shift cells, and shift reticulocytes. Archives of Pathology \& Laboratory Medicine. 1985; 109(4): 325-329. 